\title{
lodobenzene Dicarboxylates as Transferrable Oxygen Sources: Synthesis of $\alpha$-Oxygenated Ketones from Terminal Aryl Alkynes
}

\author{
Bao-Yi Ren, ${ }^{*, a, b}$ Daokun Zhong, ${ }^{a}$ Nan Guo, ${ }^{a}$ Weikun Duan, ${ }^{a}$ Shuai Song, ${ }^{a}$ and Xue Yang ${ }^{b}$ \\ ${ }^{a}$ Key Laboratory of Inorganic Molecule-Based Chemistry of Liaoning Province, \\ Shenyang University of Chemical Technology, Shenyang, Liaoning 110142, China \\ ${ }^{b}$ Department of Chemistry, Northeast Normal University, Changchun, Jilin 130024, China
}

\begin{abstract}
A facile and efficient method for preparation of $\alpha$-oxygenated ketones has been developed via the reaction of terminal aryl alkynes with various iodobenzene dicarboxylates. When bis(trifluoroacetoxy)iodobenzene (PIFA) was used, $\alpha$-hydroxy ketones were obtained in high to excellent yields. The hypervalent iodine(III) reagents can function as an electrophile, transferrable $O$-nucleophile, and an excellent leaving group. The protocol represents a direct, atom-efficient and metal-free conversion of alkynes into $\alpha$-oxygenated ketones under mild conditions.
\end{abstract}

Keywords iodobenzene dicarboxylates, transferrable oxygen sources, $\alpha$-oxygenated ketones

\section{Introduction}

$\alpha$-Oxygenated ketones, including $\alpha$-hydroxy ketones, are common structural motifs in a variety of natural products and represent one class of important intermediates in the synthesis of biologically active compounds and pharmaceuticals. To date, many procedures have been developed to prepare $\alpha$-oxygenated ketones, such as $\alpha$-oxygenation of enolates, ${ }^{[1]}$ epoxidation, ${ }^{[2]}$ dihydroxylation, ${ }^{[3]}$ aminoxylation ${ }^{[4]}$ of preformed enol ethers, enolates and enamines, and reduction of diketones. ${ }^{[5]}$

In the past decade, the application of hypervalent iodine(III) reagents in organic synthesis has attracted intense interest due to their intriguing oxidizing properties, low toxicity, mild reactivity, ease of handling and ready availability ${ }^{[6]}$ A number of reports have demonstrated the feasibility of iodobenzene diacetate-mediated alkene dioxygenation in the presence or absence of external catalysts ${ }^{[7,8]}$ and $\alpha$-oxygenation of ketones. ${ }^{[9,10]}$ However, the reaction of iodobenzene diacetate with alkynes is less well-documented. ${ }^{[11]}$ In 2009, Hou and co-workers ${ }^{[12]}$ reported the reaction of alkynes and iodobenzene diacetate, giving rise to useful $\alpha$-acetoxy ketones. In this literature, the acetic acid was used as solvent, and the temperature of reaction was at $70{ }^{\circ} \mathrm{C}$. When compared with the Au-catalyzed transformation of alkynes into $\alpha$-oxygenated ketones, ${ }^{[13]}$ the method has the distinct advantage of avoiding the utilization of an expensive and toxic transition-metal catalyst. In our research on halogen-mediated organic reactions, we found that this type of reaction can proceed efficiently in the absence of an external acetic acid catalyst and the reaction temperature can be decreased to room temperature (Scheme 1). The modified conditions developed by our group appear to be much milder and thus more attractive and practical relative to previous research. ${ }^{[11-13]}$ Through detailed study, we found that various $\alpha$-acyloxy ketones can be obtained by the reaction of alkynes with a series of iodobenzene dicarboxylates $\left[\mathrm{PhI}(\mathrm{OCOR})_{2}, \mathrm{R}=\mathrm{Me}, t\right.$-Bu, and $\left.\mathrm{Ph}\right]$. Moreover, when bis(trifluoroacetoxy)iodobenzene (PIFA) was used, $\alpha$-hydroxy ketones were obtained in high to excellent yields. The experimental results demonstrate that $\mathrm{PhI}(\mathrm{OCOR})_{2}$ itself may serve as a transferrable oxygen source as well as an electrophile and excellent leaving group. ${ }^{[14]}$

Scheme 1 Synthesis of $\alpha$-oxygenated ketones from terminal aryl alkynes.

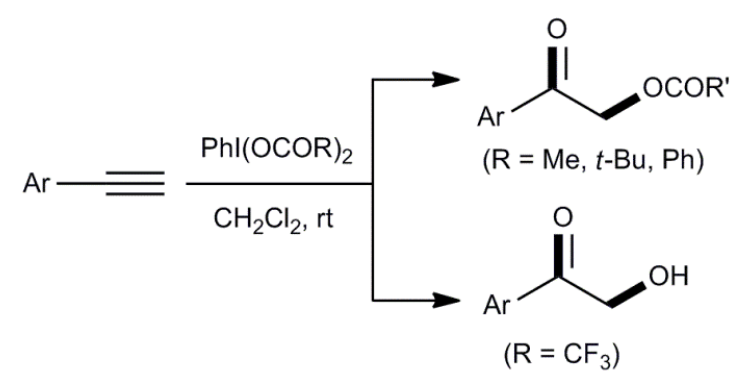

\section{Experimental}

\section{General}

All reagents were purchased from Acros, Aldrich

* E-mail:baoyir@126.com

Received December 22, 2015; accepted January 15, 2016; published online January 25, 2016.

Supporting information for this article is available on the WWW under http://www.genchemisty.org 
Chemical Co., Aladdin Industrial and Sinopharm Chemical Reagents Co. They were used without further treatment. The products were purified by column chromatography over silica gel. ${ }^{1} \mathrm{H}$ NMR and ${ }^{13} \mathrm{C}$ NMR spectra were recorded at $25{ }^{\circ} \mathrm{C}$ on a Varian $500 \mathrm{MHz}$ and $125 \mathrm{MHz}$, respectively (TMS as internal standard). IR spectra (KBr) were recorded on a Magna-560 FTIR spectrophotometer in the range of $400-4000 \mathrm{~cm}^{-1}$. Elemental analyses were measured on an E-2400 analyzer (Perkin-Elmer). Mass spectra were recorded on Agilient 1100 LCMsD mass spectrometer.

\section{General procedure for preparation of compounds} 2-7 (2a as an example)

To a solution of phenylacetylene 1a $(0.11 \mathrm{~mL}, 1.0$ mmol) in $\mathrm{CH}_{2} \mathrm{Cl}_{2}(2.0 \mathrm{~mL})$ was added $\mathrm{PhI}(\mathrm{OAc})_{2}(384$ $\mathrm{mg}, 1.2 \mathrm{mmol}$ ). The mixture was stirred at room temperature for $12 \mathrm{~h}$. The reaction mixture was poured into water and then extracted with $\mathrm{CH}_{2} \mathrm{Cl}_{2}(3 \times 10 \mathrm{~mL})$. The combined organic phase was washed with water $(3 \times 10$ $\mathrm{mL}$ ), filtered and concentrated under reduced pressure. The crude product was purified by flash chromatography [silica gel, $V$ (petroleum ether $): V($ ethyl acetate $)=$ $3: 1]$ to give $\mathbf{2 a}(162 \mathrm{mg}, 91 \%)$ as a white solid.

\section{Characterization of compounds $2-7$}

2-Oxo-2-phenylethyl acetate (2a): White solid, m.p. $38-40{ }^{\circ} \mathrm{C} ;{ }^{\mathrm{H}} \mathrm{NMR}\left(500 \mathrm{MHz}, \mathrm{CDCl}_{3}\right) \delta: 2.23(\mathrm{~s}, 3 \mathrm{H})$, $5.32(\mathrm{~s}, 2 \mathrm{H}), 7.47-7.50(\mathrm{~m}, 2 \mathrm{H}), 7.59-7.62(\mathrm{~m}, 1 \mathrm{H})$, 7.90-7.92 (m, 2H); ${ }^{13} \mathrm{C}$ NMR $\left(125 \mathrm{MHz}, \mathrm{CDCl}_{3}\right) \delta$ : 20.5, 66.0, 127.7, 128.8, 133.9, 134.1, 170.4, 192.1; IR (KBr) v: 3415, 2922, 2845, 2353, 1747, 1695, 1383, 1217, 1052, $679 \mathrm{~cm}^{-1}$; MS m/z (\%): $179.2(\mathrm{M}+\mathrm{H}, 100)$. Anal. calcd for $\mathrm{C}_{10} \mathrm{H}_{10} \mathrm{O}_{3}$ : C 67.41, $\mathrm{H}$ 5.66; found $\mathrm{C}$ 67.68, H 5.63 .

2-Oxo-2-( $p$-tolyl)ethyl acetate (2b): White solid, m.p. $81-83{ }^{\circ} \mathrm{C} ;{ }^{1} \mathrm{H}$ NMR $\left(500 \mathrm{MHz}, \mathrm{CDCl}_{3}\right) \delta: 2.23(\mathrm{~s}, 3 \mathrm{H})$, $2.42(\mathrm{~s}, 3 \mathrm{H}), 5.32(\mathrm{~s}, 2 \mathrm{H}), 7.28(\mathrm{~d}, J=8.5 \mathrm{~Hz}, 2 \mathrm{H}), 7.81$ $(\mathrm{d}, J=8.0 \mathrm{~Hz}, 2 \mathrm{H}) ;{ }^{13} \mathrm{C}$ NMR $\left(125 \mathrm{MHz}, \mathrm{CDCl}_{3}\right) \delta$ : $20.5,21.7,65.9,127.8,129.5,131.7,144.8,170.4$, 191.7; IR (KBr) v: 3041, 2954, 1749, 1695, 1604, 1573, 1241, $810 \mathrm{~cm}^{-1}$; MS m/z (\%): $193.1(\mathrm{M}+\mathrm{H}, 100)$. Anal. calcd for $\mathrm{C}_{11} \mathrm{H}_{12} \mathrm{O}_{3}$ : C 68.74, H 6.29; found $\mathrm{C} 68.55, \mathrm{H}$ 6.27 .

2-Oxo-2-( $m$-tolyl)ethyl acetate (2c): Yellow oil; ${ }^{1} \mathrm{H}$ NMR $\left(500 \mathrm{MHz}, \mathrm{CDCl}_{3}\right) \delta: 2.23$ (s, 3H), 2.41 (s, $3 \mathrm{H}), 5.33(\mathrm{~s}, 2 \mathrm{H}), 7.37(\mathrm{~d}, J=7.5 \mathrm{~Hz}, 1 \mathrm{H}), 7.41(\mathrm{~s}, 1 \mathrm{H})$, $7.72(\mathrm{t}, J=7.5 \mathrm{~Hz}, 2 \mathrm{H}) ;{ }^{13} \mathrm{C}$ NMR $\left(125 \mathrm{MHz}, \mathrm{CDCl}_{3}\right) \delta$ : $20.5,21.2,66.0,124.8,128.1,128.6,134.1,134.6$, 138.6, 170.4, 192.2; IR (KBr) v: 2965, 2928, 1751, 1698 , 1612, 1369, 1232, 1087, 959, 806, 781, 605, $560 \mathrm{~cm}^{-1}$; MS $m / z(\%): 193.2(\mathrm{M}+\mathrm{H}, 100)$. Anal. calcd for $\mathrm{C}_{11} \mathrm{H}_{12} \mathrm{O}_{3}$ : C 68.74, H 6.29; found C 68.57, H 6.26.

2-(4-(Tert-butyl)phenyl)-2-oxoethyl acetate (2d): Yellow oil; ${ }^{1} \mathrm{H}$ NMR $\left(500 \mathrm{MHz}, \mathrm{CDCl}_{3}\right) \delta: 1.34(\mathrm{~s}, 9 \mathrm{H})$, $2.24(\mathrm{~s}, 3 \mathrm{H}), 5.34(\mathrm{~s}, 2 \mathrm{H}), 7.50(\mathrm{~d}, J=8.5 \mathrm{~Hz}, 2 \mathrm{H}), 7.86$ $(\mathrm{d}, J=8.5 \mathrm{~Hz}, 2 \mathrm{H}) ;{ }^{13} \mathrm{C}$ NMR $\left(125 \mathrm{MHz}, \mathrm{CDCl}_{3}\right) \delta$ :
20.6, 31.0, 31.1, 35.2, 65.9, 125.8, 127.7, 131.6, 157.7, 170.4, 191.7; IR (KBr) v: 2972, 2865, 1769, 1692, 1583, 1231, 1084, 987, 841, 734, 639, $581 \mathrm{~cm}^{-1}$; MS $m / z(\%)$ : $235.3(\mathrm{M}+\mathrm{H}, 100)$. Anal. calcd for $\mathrm{C}_{14} \mathrm{H}_{18} \mathrm{O}_{3}: \mathrm{C} 71.77$, $\mathrm{H}$ 7.74; found C 71.63, H 7.71.

2-(4-Methoxyphenyl)-2-oxoethyl acetate (2e): White solid, m.p. 53-55 ${ }^{\circ} \mathrm{C} ;{ }^{1} \mathrm{H}$ NMR $\left(500 \mathrm{MHz}, \mathrm{CDCl}_{3}\right) \delta$ : $2.21(\mathrm{~s}, 3 \mathrm{H}), 3.85(\mathrm{~s}, 3 \mathrm{H}), 5.29(\mathrm{~s}, 2 \mathrm{H}), 6.93(\mathrm{~d}, J=8.5$ $\mathrm{Hz}, 2 \mathrm{H}), 7.88(\mathrm{~d}, J=9.0 \mathrm{~Hz}, 2 \mathrm{H}) ;{ }^{13} \mathrm{C}$ NMR $(125 \mathrm{MHz}$, $\left.\mathrm{CDCl}_{3}\right) \delta: 20.5,55.5,65.7,114.0,127.2,130.0,164.0$, 170.4, 190.6; IR (KBr) v: 2928, 2837, 1755, 1690, 1587, 1436, 1203, 1169, 1088, 953, 828, 775, 608, $564 \mathrm{~cm}^{-1}$; MS $m / z(\%): 209.1(\mathrm{M}+\mathrm{H}, 100)$. Anal. calcd for $\mathrm{C}_{11} \mathrm{H}_{12} \mathrm{O}_{4}$ : C 63.45, H 5.81; found C 63.24, H 5.79.

2-(4-Fluorophenyl)-2-oxoethyl acetate (2f): White solid, m.p. 46-48 ${ }^{\circ} \mathrm{C} ;{ }^{1} \mathrm{H}$ NMR $\left(500 \mathrm{MHz}, \mathrm{CDCl}_{3}\right) \delta$ : $2.23(\mathrm{~s}, 3 \mathrm{H}), 5.31(\mathrm{~s}, 2 \mathrm{H}), 7.15-7.18(\mathrm{~m}, 2 \mathrm{H}), 7.94-$ $7.97(\mathrm{~m}, 2 \mathrm{H}) ;{ }^{13} \mathrm{C}$ NMR $\left(125 \mathrm{MHz}, \mathrm{CDCl}_{3}\right) \delta: 20.5$, $65.8,116.0,116.1,130.4,130.5,130.6,130.6,165.1$, 167.1, 170.3, 190.6; IR (KBr) v: 3074, 2941, 1746, 1699, 1601, 1509, 1246, $833 \mathrm{~cm}^{-1}$; MS m/z (\%): $197.3(\mathrm{M}+\mathrm{H}$, 100). Anal. calcd for $\mathrm{C}_{10} \mathrm{H}_{9} \mathrm{FO}_{3}$ : C 61.22, $\mathrm{H} 4.62$; found C 61.17, H 4.60.

2-(3-Chlorophenyl)-2-oxoethyl acetate (2g): Yellow oil; ${ }^{1} \mathrm{H}$ NMR $\left(500 \mathrm{MHz}, \mathrm{CDCl}_{3}\right) \delta: 2.23(\mathrm{~s}, 3 \mathrm{H}), 5.30(\mathrm{~s}$, $2 \mathrm{H}), 7.45(\mathrm{t}, J=8.0 \mathrm{~Hz}, 1 \mathrm{H}), 7.58-7.59(\mathrm{~m}, 1 \mathrm{H}), 7.78$ $(\mathrm{d}, J=7.5 \mathrm{~Hz}, 1 \mathrm{H}), 7.89$ (s, $1 \mathrm{H}) ;{ }^{13} \mathrm{C}$ NMR $(125 \mathrm{MHz}$, $\left.\mathrm{CDCl}_{3}\right) \delta: 20.4,65.8,125.7,127.8,130.1,133.8,135.2$, 135.5, 170.3, 191.0; IR (KBr) v: 3038, 2904, 1761, 1735, 1573, 1438, 1372, 1209, 1090, 852, 786, 744, 686, 586 $\mathrm{cm}^{-1}$; MS $\mathrm{m} / z(\%): 214.0(\mathrm{M}+\mathrm{H}, 100)$. Anal. calcd for $\mathrm{C}_{10} \mathrm{H}_{9} \mathrm{ClO}_{3}$ : C 56.49, H 4.27; found C 56.25, H 4.25.

2-Oxo-2-(thiophen-2-yl)ethyl acetate (2h): Brown oil; ${ }^{1} \mathrm{H}$ NMR $\left(500 \mathrm{MHz}, \mathrm{CDCl}_{3}\right) \delta: 2.23(\mathrm{~s}, 3 \mathrm{H}), 5.23(\mathrm{~s}$, 2H), $7.17(\mathrm{t}, J=4.5 \mathrm{~Hz}, 1 \mathrm{H}), 7.71(\mathrm{~d}, J=4.5 \mathrm{~Hz}, 1 \mathrm{H})$, $7.76(\mathrm{~d}, J=4.5 \mathrm{~Hz}, 1 \mathrm{H}) ;{ }^{13} \mathrm{C}$ NMR $\left(125 \mathrm{MHz}, \mathrm{CDCl}_{3}\right) \delta$ : 20.5, 65.7, 128.2, 131.9, 134.3, 140.3, 170.3, 185.4; IR (KBr) v: 3012, 1739, 1685, 1483, 1290, 817, 694, 597 $\mathrm{cm}^{-1}$; MS $\mathrm{m} / z(\%): 185.2(\mathrm{M}+\mathrm{H}, 100)$. Anal. calcd for $\mathrm{C}_{8} \mathrm{H}_{8} \mathrm{O}_{3} \mathrm{~S}$ : C 52.16, H 4.38; found C 52.43, H 4.39.

2-(4-Ethynylphenyl)-2-oxoethyl acetate (2i): Yellow solid, m.p. 89-91 ${ }^{\circ} \mathrm{C} ;{ }^{1} \mathrm{H}$ NMR $\left(500 \mathrm{MHz}, \mathrm{CDCl}_{3}\right) \delta$ : $2.23(\mathrm{~s}, 3 \mathrm{H}), 3.29(\mathrm{~s}, 1 \mathrm{H}), 5.32(\mathrm{~s}, 2 \mathrm{H}), 7.59$ (d, $J=9.0$ $\mathrm{Hz}, 2 \mathrm{H}), 7.87(\mathrm{t}, J=4.5 \mathrm{~Hz}, 2 \mathrm{H}) ;{ }^{13} \mathrm{C}$ NMR $(125 \mathrm{MHz}$, $\left.\mathrm{CDCl}_{3}\right) \delta: 20.5,65.9,81.0,82.5,127.6,127.7,132.5$, 133.8, 170.3, 191.4; IR (KBr) v: 3272, 3070, 2943, 2107, 1744, 1702, 1604, 1557, 1219, $822 \mathrm{~cm}^{-1}$; MS m/z (\%): 203.2 (M+H, 100). Anal. calcd for $\mathrm{C}_{12} \mathrm{H}_{10} \mathrm{O}_{3}: \mathrm{C} 71.28$, $\mathrm{H}$ 4.98; found C 71.45, H 4.95 .

2-Hydroxy-1-phenylethanone (3a): White solid, m.p. $81-83{ }^{\circ} \mathrm{C} ;{ }^{1} \mathrm{H}$ NMR $\left(500 \mathrm{MHz}, \mathrm{CDCl}_{3}\right) \delta: 3.53(\mathrm{~s}, 1 \mathrm{H})$, $4.89(\mathrm{~s}, 2 \mathrm{H}), 7.51(\mathrm{t}, J=8.0 \mathrm{~Hz}, 2 \mathrm{H}), 7.86-7.88(\mathrm{~m}$, $1 \mathrm{H}), 7.92(\mathrm{t}, J=4.5 \mathrm{~Hz}, 2 \mathrm{H}) ;{ }^{13} \mathrm{C}$ NMR $(125 \mathrm{MHz}$, $\left.\mathrm{CDCl}_{3}\right) \delta: 65.4,127.6,128.9,133.2,134.3,198.3 . \mathrm{IR}$ (KBr) $v: 3417,3385,1677,1432,1405,1229,1095,971$, 761, 689, $617 \mathrm{~cm}^{-1}$; MS m/z (\%): $137.2(\mathrm{M}+\mathrm{H}, 100)$. Anal. calcd for $\mathrm{C}_{8} \mathrm{H}_{8} \mathrm{O}_{2}$ : C 70.57, $\mathrm{H}$ 5.92; found $\mathrm{C} 70.32$, H 5.94 . 
2-Hydroxy-1-(p-tolyl)ethanone (3b): White solid, m.p. $71-73{ }^{\circ} \mathrm{C} ;{ }^{1} \mathrm{H}$ NMR $\left(500 \mathrm{MHz}, \mathrm{CDCl}_{3}\right) \delta: 2.44$ (s, $3 \mathrm{H}), 5.56(\mathrm{~s}, 2 \mathrm{H}), 7.32(\mathrm{~d}, J=8.5 \mathrm{~Hz}, 2 \mathrm{H}), 7.80(\mathrm{~d}, J=$ $8.0 \mathrm{~Hz}, 2 \mathrm{H}) ;{ }^{13} \mathrm{C} \mathrm{NMR}\left(125 \mathrm{MHz}, \mathrm{CDCl}_{3}\right) \delta: 21.8,65.2$, 127.7, 129.6, 145.3, 197.9; IR (KBr) v: 3439, 2973, 1680, 1612, 1416, 1288, 1165, 965, $754 \mathrm{~cm}^{-1}$; MS $m / z(\%): 151.2(\mathrm{M}+\mathrm{H}, 100)$. Anal. calcd for $\mathrm{C}_{9} \mathrm{H}_{10} \mathrm{O}_{2}$ : C 71.98, H 6.71; found C 71.75, H 6.72.

2-Hydroxy-1-( $m$-tolyl)ethanone (3c): White solid, m.p. $57-60{ }^{\circ} \mathrm{C} ;{ }^{1} \mathrm{H}$ NMR $\left(500 \mathrm{MHz}, \mathrm{CDCl}_{3}\right) \delta: 2.43$ (s, $3 \mathrm{H}), 5.56(\mathrm{~s}, 2 \mathrm{H}), 7.41(\mathrm{t}, J=7.5 \mathrm{~Hz}, 1 \mathrm{H}), 7.46$ (d, $J=$ $7.5 \mathrm{~Hz}, 1 \mathrm{H}), 7.69(\mathrm{t}, J=8.0 \mathrm{~Hz}, 2 \mathrm{H}) .{ }^{13} \mathrm{C}$ NMR $(125$ $\left.\mathrm{MHz}, \mathrm{CDCl}_{3}\right) \delta: 21.2,65.3,124.8,127.3,128.3,130.6$, 134.4, 135.0, 198.5; IR (KBr) v: 3450, 2898, 1687, 1293 , 1089, 1032, 985, 774, $682 \mathrm{~cm}^{-1}$; MS m/z (\%): $151.1(\mathrm{M}$ $+\mathrm{H}, 100)$. Anal. calcd for $\mathrm{C}_{9} \mathrm{H}_{10} \mathrm{O}_{2} \mathrm{C}$ 71.98, H 6.71; found $\mathrm{C} 71.87, \mathrm{H} 6.73$.

1-(4-(t-Butyl)phenyl)-2-hydroxyethanone (3d): Yellow oil; ${ }^{1} \mathrm{H}$ NMR $\left(500 \mathrm{MHz}, \mathrm{CDCl}_{3}\right) \delta: 1.35(\mathrm{~s}, 9 \mathrm{H})$, $5.57(\mathrm{~s}, 2 \mathrm{H}), 7.53(\mathrm{~d}, J=8.5 \mathrm{~Hz}, 2 \mathrm{H}), 7.85(\mathrm{~d}, J=8.5$ $\mathrm{Hz}, 2 \mathrm{H}) ;{ }^{13} \mathrm{C}$ NMR $\left(125 \mathrm{MHz}, \mathrm{CDCl}_{3}\right) \delta: 30.9,35.1$, 65.2, 125.4, 127.6, 130.0, 157.4, 197.9; IR (KBr) v: $3420,2971,1648,1505,1475,1381,1225,937,853$, $796 \mathrm{~cm}^{-1}$; MS $m / z(\%): 193.3(\mathrm{M}+\mathrm{H}, 100)$. Anal. calcd for $\mathrm{C}_{12} \mathrm{H}_{16} \mathrm{O}_{2}$ : C 74.97, $\mathrm{H} \mathrm{8.39}$; found $\mathrm{C} 74.75, \mathrm{H} 8.36$.

2-Hydroxy-1-(4-methoxyphenyl)ethanone

(3e): White solid, m.p. $64-66{ }^{\circ} \mathrm{C} ;{ }^{1} \mathrm{H}$ NMR $(500 \mathrm{MHz}$, $\left.\mathrm{CDCl}_{3}\right) \delta: 3.89(\mathrm{~s}, 3 \mathrm{H}), 5.53(\mathrm{~s}, 2 \mathrm{H}), 6.98-7.00(\mathrm{~m}$, $2 \mathrm{H}), 7.87-7.89(\mathrm{~m}, 2 \mathrm{H}) ;{ }^{13} \mathrm{C}$ NMR $\left(125 \mathrm{MHz}, \mathrm{CDCl}_{3}\right)$ $\delta$ : $55.5,67.9,114.2,126.2,130.1,164.4,187.1$; IR (KBr): 3345, 3027, 2981, 2944, 1688, 1602, 1515, 1468, 1382, 1026, $829 \mathrm{~cm}^{-1}$; MS m/z (\%): $166.1(\mathrm{M}+\mathrm{H}, 100)$. Anal. calcd for $\mathrm{C}_{9} \mathrm{H}_{10} \mathrm{O}_{3}: \mathrm{C} 65.05, \mathrm{H} 6.07$; found $\mathrm{C}$ 65.24, H 6.04.

1-(4-Fluorophenyl)-2-hydroxyethanone (3f): White solid, m.p. 96-98 ${ }^{\circ} \mathrm{C} ;{ }^{1} \mathrm{H}$ NMR $\left(500 \mathrm{MHz}, \mathrm{CDCl}_{3}\right) \delta$ : $5.55(\mathrm{~s}, 2 \mathrm{H}), 7.21(\mathrm{t}, J=8.5 \mathrm{~Hz}, 2 \mathrm{H}), 7.93-7.96(\mathrm{~m}$, $2 \mathrm{H}) ;{ }^{13} \mathrm{C} \mathrm{NMR}\left(125 \mathrm{MHz}, \mathrm{CDCl}_{3}\right) \delta: 65.3,116.3,129.8$, 130.4, 130.5, 167.4, 196.8; IR (KBr) v: 3391, 3072, 2936, 1685, 1597, 1508, $838 \mathrm{~cm}^{-1}$; MS $m / z(\%): 155.1$ $(\mathrm{M}+\mathrm{H}, 100)$. Anal. calcd for $\mathrm{C}_{8} \mathrm{H}_{7} \mathrm{FO}_{2}: \mathrm{C} 62.34, \mathrm{H} 4.58$; found C 62.13, $\mathrm{H} 4.54$.

1-(3-Chlorophenyl)-2-hydroxyethanone (3g): White solid, m.p. 89-91 ${ }^{\circ} \mathrm{C} ;{ }^{1} \mathrm{H}$ NMR $\left(500 \mathrm{MHz}, \mathrm{CDCl}_{3}\right) \delta$ : $5.54(\mathrm{~s}, 2 \mathrm{H}), 7.48(\mathrm{t}, J=8.0 \mathrm{~Hz}, 1 \mathrm{H}), 7.62-7.64(\mathrm{~m}$, $1 \mathrm{H}), 7.77(\mathrm{~d}, J=8.0 \mathrm{~Hz}, 1 \mathrm{H}), 7.87(\mathrm{t}, J=2.0 \mathrm{~Hz}, 1 \mathrm{H})$; ${ }^{13} \mathrm{C}$ NMR $\left(125 \mathrm{MHz}, \mathrm{CDCl}_{3}\right) \delta: 59.4,128.4,129.6$, $129.8,130.3,134.1,134.7,171.3$; IR $(\mathrm{KBr}) v: 3432$, $3375,1684,1607,1412,1227,1164,1106,981,837$, 602, $568 \mathrm{~cm}^{-1}$; MS m/z (\%): $172.1(\mathrm{M}+\mathrm{H}, 100)$. Anal. calcd for $\mathrm{C}_{8} \mathrm{H}_{7} \mathrm{ClO}_{2}$ : C 56.32, $\mathrm{H} 4.14$; found $\mathrm{C} 56.02, \mathrm{H}$ 4.11.

2-Hydroxy-1-(thiophen-2-yl)ethanone (3h): Brown oil; ${ }^{1} \mathrm{H}$ NMR $\left(500 \mathrm{MHz}, \mathrm{CDCl}_{3}\right) \delta: 4.80(\mathrm{~s}, 2 \mathrm{H}), 7.74(\mathrm{t}$, $J=2.5 \mathrm{~Hz}, 3 \mathrm{H}) ;{ }^{13} \mathrm{C}$ NMR $\left(125 \mathrm{MHz}, \mathrm{CDCl}_{3}\right) \delta: 65.4$, 128.4, 132.0, 134.5, 139.3, 191.2; IR (KBr) v: 3432, 3187, 1676, 1533, 1421, 1280, 1072, 943, 864, 720, 589 $\mathrm{cm}^{-1}$; MS m/z (\%): $143.1(\mathrm{M}+\mathrm{H}, 100)$. Anal. calcd for
$\mathrm{C}_{6} \mathrm{H}_{6} \mathrm{O}_{2} \mathrm{~S}$ : C 50.69, H 4.25; found C 50.37, H 4.29.

1-(4-Ethynylphenyl)-2-hydroxyethanone (3j): Yellow solid, m.p. $103-105{ }^{\circ} \mathrm{C} ;{ }^{1} \mathrm{H}$ NMR $(500 \mathrm{MHz}$, $\left.\mathrm{CDCl}_{3}\right) \delta: 3.32(\mathrm{~s}, 1 \mathrm{H}), 5.56(\mathrm{~s}, 2 \mathrm{H}), 7.62(\mathrm{~d}, J=8.5 \mathrm{~Hz}$, $2 \mathrm{H}), 7.86(\mathrm{~d}, J=8.5 \mathrm{~Hz}, 2 \mathrm{H}) ;{ }^{13} \mathrm{C}$ NMR $(125 \mathrm{MHz}$, $\left.\mathrm{CDCl}_{3}\right) \delta: 68.1,81.1,81.5,127.6,127.7,132.6,132.7$, 188.1; IR (KBr) v: 3432, 3296, 2125, 1686, 1287, 1237 , $1125,953,817,565 \mathrm{~cm}^{-1}$; MS $m / z(\%): 161.1(\mathrm{M}+\mathrm{H}$, 100). Anal. calcd for $\mathrm{C}_{10} \mathrm{H}_{8} \mathrm{O}_{2}$ : C 74.99, H 5.03; found C 74.68, H 5.07.

2-Oxo-2-phenylethyl pivalate (4): White solid, m.p. $58-60{ }^{\circ} \mathrm{C} ;{ }^{\mathrm{H}} \mathrm{NMR}\left(500 \mathrm{MHz}, \mathrm{CDCl}_{3}\right) \delta: 1.31(\mathrm{~s}, 9 \mathrm{H})$, $5.32(\mathrm{~s}, 2 \mathrm{H}), 7.48(\mathrm{t}, J=8.0 \mathrm{~Hz}, 2 \mathrm{H}), 7.59(\mathrm{t}, J=7.5 \mathrm{~Hz}$, $1 \mathrm{H}), 7.90(\mathrm{t}, J=7.0 \mathrm{~Hz}, 2 \mathrm{H}) ;{ }^{13} \mathrm{C}$ NMR $(125 \mathrm{MHz}$, $\left.\mathrm{CDCl}_{3}\right) \delta: 27.1,38.7,65.8,127.7,128.7,133.7,134.3$, 177.9, 192.4; IR (KBr) v: 2933, 1737, 1697, 1596, 1576, 1395, 1370, 1149, 688, $669 \mathrm{~cm}^{-1}$; MS m/z (\%): 221.2 $(\mathrm{M}+\mathrm{H}, 100)$. Anal. calcd for $\mathrm{C}_{13} \mathrm{H}_{16} \mathrm{O}_{3}: \mathrm{C} 70.89, \mathrm{H}$ 7.32; found C 70.63, H 7.35.

2-Oxo-2-phenylethyl benzoate (5): White solid, m.p. $91-93{ }^{\circ} \mathrm{C} ;{ }^{1} \mathrm{H}$ NMR $\left(500 \mathrm{MHz}, \mathrm{CDCl}_{3}\right) \delta: 5.58(\mathrm{~s}, 2 \mathrm{H})$, $7.46-7.52(\mathrm{~m}, 4 \mathrm{H}), 7.58-7.62(\mathrm{~m}, 2 \mathrm{H}), 7.97(\mathrm{t}, J=6.0$ $\mathrm{Hz}, 2 \mathrm{H}), 8.14-8.15(\mathrm{~m}, 2 \mathrm{H}) ;{ }^{13} \mathrm{C}$ NMR $(125 \mathrm{MHz}$, $\left.\mathrm{CDCl}_{3}\right) \delta: 66.4,127.8,128.4,128.5,128.8,129.9,130.1$, 133.3, 133.7, 133.8, 166.0, 192.0; IR (KBr) v: 3057 , 2944, 1718, 1697, 1597, 1581, 1283, 713, $689 \mathrm{~cm}^{-1}$; MS $m / z(\%): 241.1(\mathrm{M}+\mathrm{H}, 100)$. Anal. calcd for $\mathrm{C}_{15} \mathrm{H}_{12} \mathrm{O}_{3}$ : C 74.99, H 5.03; found C 74.68, H 5.05.

2-Oxo-2-phenylethane-1,1-diyl bis(4-methylbenzenesulfonate) (6): White solid, m.p. 87-89 ${ }^{\circ} \mathrm{C} ;{ }^{1} \mathrm{H}$ NMR $\left(500 \mathrm{MHz}, \mathrm{CDCl}_{3}\right) \delta: 2.44(\mathrm{~s}, 6 \mathrm{H}), 6.96(\mathrm{~s}, 1 \mathrm{H}), 7.28(\mathrm{~d}$, $J=8.5 \mathrm{~Hz}, 4 \mathrm{H}), 7.43-7.47(\mathrm{~m}, 2 \mathrm{H}), 7.59-7.62(\mathrm{~m}$, $1 \mathrm{H}), 7.72(\mathrm{~d}, J=8.5 \mathrm{~Hz}, 4 \mathrm{H}), 7.90-7.92(\mathrm{~m}, 2 \mathrm{H}) ;{ }^{13} \mathrm{C}$ NMR $\left(125 \mathrm{MHz}, \mathrm{CDCl}_{3}\right) \delta$ : 195.2, 145.5,141.4, 136.0, 134.2, 133.1, 130.5, 129.2, 128.6, 103.7, 22.8; IR (KBr) $v: 3047,2989,1692,1608,1433,1348,1274,987,802$, 699, $576 \mathrm{~cm}^{-1}$; MS m/z (\%): $461.5(\mathrm{M}+\mathrm{H}, 100)$. Anal. calcd for $\mathrm{C}_{22} \mathrm{H}_{20} \mathrm{O}_{7} \mathrm{~S}_{2}$ : C 57.38, H 4.38; found C 57.46, H 4.35 .

2-Oxo-2-phenylethyl butyrate (7): Yellow oil; ${ }^{1} \mathrm{H}$ $\operatorname{NMR}\left(500 \mathrm{MHz}, \mathrm{CDCl}_{3}\right) \delta: 0.97-1.01(\mathrm{~m}, 3 \mathrm{H}), 1.69-$ $1.77(\mathrm{~m}, 2 \mathrm{H}), 2.47-2.50(\mathrm{~m}, 2 \mathrm{H}), 5.35(\mathrm{~s}, 2 \mathrm{H}), 7.48(\mathrm{t}$, $J=7.5 \mathrm{~Hz}, 2 \mathrm{H}), 7.60(\mathrm{~d}, J=7.5 \mathrm{~Hz}, 1 \mathrm{H}), 7.92(\mathrm{~d}, J=$ $8.0 \mathrm{~Hz}, 2 \mathrm{H}) ;{ }^{3} \mathrm{C}$ NMR $\left(125 \mathrm{MHz}, \mathrm{CDCl}_{3}\right) \delta: 193.7$, 175.1, 135.5, 133.2, 129.2, 128.6, 68.7, 35.8, 19.2, 13.3; IR (KBr) v: 3047, 2978, 2843, 1740, 1421, 1335, 1244, 1196, 1075, 984, 789, $719 \mathrm{~cm}^{-1}$; MS $m / z(\%): 207.0$ (M $+\mathrm{H}, 100)$. Anal. calcd for $\mathrm{C}_{12} \mathrm{H}_{14} \mathrm{O}_{3}$ : C 69.88, H 6.84; found C 69.95, H 6.81.

\section{Results and Discussion}

Initially, the model reaction of phenylacetylene 1a and $\mathrm{PhI}(\mathrm{OAc})_{2}$ was examined under various conditions (Table 1). The reactions of $\mathbf{1 a}$ with 1.2 equiv. of $\mathrm{PhI}(\mathrm{OAc})_{2}$ were performed at room temperature in solvents such as DMF, MeCN, THF and toluene. $\alpha$-Acetoxy ketone $2 \mathrm{a}$ was obtained in $54 \%-81 \%$ yield 
(Table 1, Entries 1-4). Halogenated solvents $\left[\mathrm{CHCl}_{3}\right.$, (dichloroethane) DCE and (dichloromethane) DCM] appear to be more suitable (Table 1, Entries 5-7) and DCM gave the best result ( $91 \%$ yields). Though HOAc was selected as the solvent, the yield exhibited no change (Table 1, Entry 8). Both raising and lowering of the temperature led to decreased yields (Table 1, Entries 9 and 10). 1.0 or 0.8 equiv. of $\mathrm{PhI}(\mathrm{OAc})_{2}$ was insufficient to drive the reaction to completion (Table 1, Entries 11 and 12). It is noteworthy that the yields exhibit almost no change when dry or wet DCM was used as the solvent.

Table 1 Optimization of the reaction conditions ${ }^{a}$

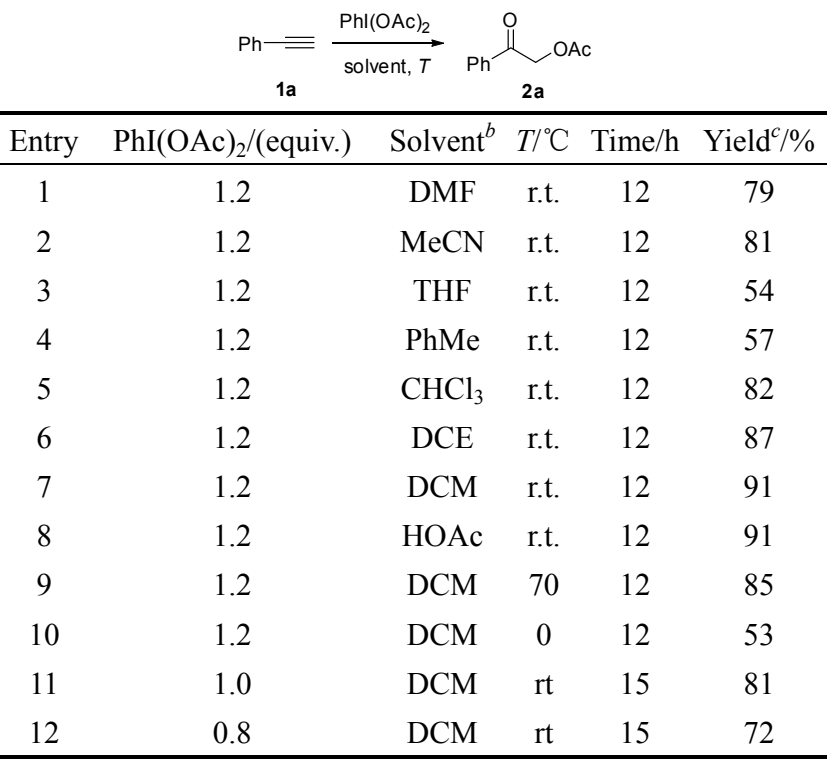

${ }^{a}$ Reactions were carried out on a $1.0 \mathrm{mmol}$ scale in $2.0 \mathrm{~mL}$ of solvent. ${ }^{b}$ No distinct difference in yield was observed when both, dry and wet solvents were used. ${ }^{c}$ Isolated yield.

Under the optimized conditions (Table 1, Entry 7), a range of reactions were carried out with various alkynes $1(1.0 \mathrm{mmol})$ and $\mathrm{PhI}(\mathrm{OAc})_{2}$ (1.2 equiv.) in DCM (2.0 $\mathrm{mL}$ ) (Table 2). The majority of the reactions proceeded efficiently to afford the corresponding $\alpha$-acetoxy ketones $(\mathbf{2} \mathbf{a}-\mathbf{2} \mathbf{h}$ and $\mathbf{2} \mathbf{j})$ in high to excellent yield. The substituents on the terminal arylacetylene may be alkyls (e.g., methyl and $t$-butyl), alkoxyls (e.g., methoxy) and halogen atoms (e.g., $\mathrm{Cl}$ and $\mathrm{F}$ ). Heteroaryls such as 2-thienyl worked well, giving acetic acid 2-oxo-2-thiophen-2-yl-ethyl ester (2h) in $87 \%$ yield, while 2-pyridylacetylene failed, probably due to the strong electron-withdrawing property of the pyridyl group. Bisacetylene can react at one of the triple bonds, affording acetylene-terminated $\alpha$-acetoxy ketone $\mathbf{2 j}$ in $89 \%$ yield. Terminal and internal alkyl alkynes were also investigated with only trace amounts of the target products being observed.
Table 2 Reaction of alkynes with $\mathrm{PhI}(\mathrm{OAc})_{2}{ }^{a}$

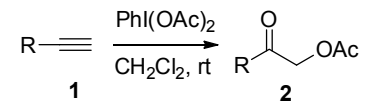

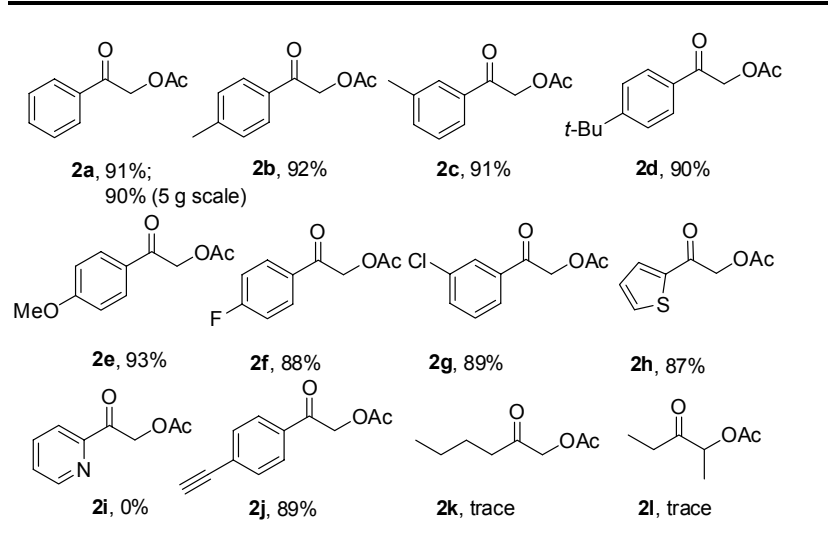

$\overline{{ }^{a} \text { Reactions were carried out with } 1(1.0 \mathrm{mmol}) \text { and } \mathrm{PhI}(\mathrm{OAc})_{2}}$ (1.2 equiv.) in DCM $(2.0 \mathrm{~mL})$ at r.t. for $12 \mathrm{~h}$. The product yields are isolated yields.

To investigate the influence of different ligands on the iodine(III) reagents, the reaction was further examined. Thus, the reaction of alkynes with PIFA was conducted under otherwise identical conditions (Table 3). As a result, instead of the formation of $\alpha$-trifluoroacetoxy ketones, a series of $\alpha$-hydroxy ketones $\mathbf{3 a}-\mathbf{3 h}$ and $\mathbf{3 j}$ were obtained in high to excellent yield $(87 \%-92 \%)$. The structure of $3 \mathbf{e}$ was confirmed by single-crystal X-ray diffraction (Figure 1) ${ }^{[15]} \mathrm{A} 5 \mathrm{~g}$ scale preparation of compounds 2a and 3a was readily achieved in excellent yields with simple purification via recrystallization of the crude product from ethanol.

Table 3 Reaction of alkynes with bis(trifluoroacetoxy)iodobenzene $^{a}$

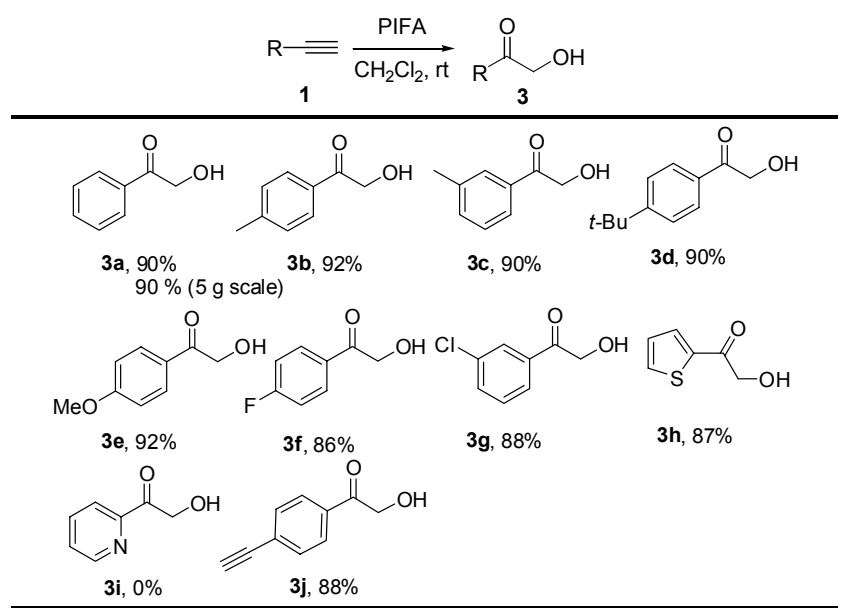

${ }^{a}$ Reactions were carried out with $1(1.0 \mathrm{mmol})$ and PIFA (1.2 equiv.) in DCM $(2.0 \mathrm{~mL})$ at r.t. for $12 \mathrm{~h}$. The product yields are isolated yields. 


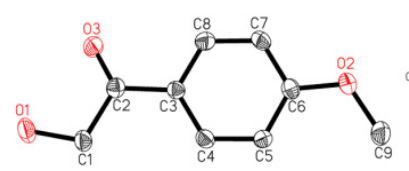

$3 \mathbf{e}$

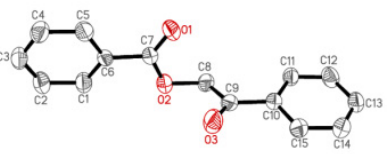

5
Figure 1 ORTEP drawings of $\mathbf{3 e}$ and $\mathbf{5}$.

The reaction was further explored by investigating other hypervalent iodine(III) reagents (Scheme 2). ${ }^{[16]}$ It was found that the reaction of $1 \mathrm{a}$ with $\mathrm{PhI}(\mathrm{OPiv})_{2}$ or $\mathrm{PhI}(\mathrm{OBz})_{2}$ furnished the corresponding $\alpha$-t-butylcarbonyloxy ketone 4 and $\alpha$-benzoyloxy ketone 5 in $86 \%$ and $89 \%$ yield, respectively [Eq. (1) and (2)]. ${ }^{[17]}$ Interestingly, Koser's reagent $[\mathrm{PhI}(\mathrm{OH})(\mathrm{OTs})]$ could also react with 1a to give $\alpha$-bistosyloxy ketone $\mathbf{6}$ in $62 \%$ yield [Eq. (3)]. ${ }^{[18,19]}$ The structure of 5 was confirmed by single-crystal X-ray diffraction (Figure 1). Clearly, the reaction was capable of introducing other types of oxygen functionality by simply modulating the ligands on the hypervalent iodine(III) reagent.

Scheme 2 Reaction of alkynes with other hypervalent iodine(III) reagents.

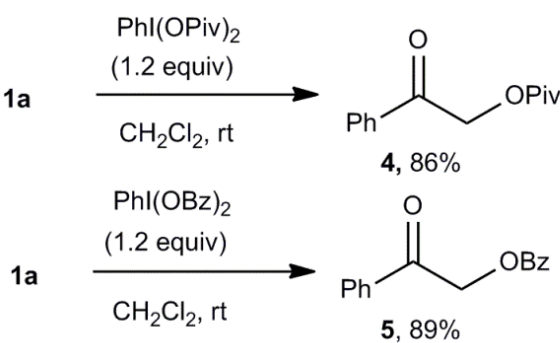

$$
\begin{aligned}
& \text { 1a } \underset{\mathrm{CH}_{2} \mathrm{Cl}_{2}, \mathrm{rt}}{\stackrel{\mathrm{Phl}(\mathrm{OH})(\mathrm{OTs})}{(1.2 \text { equiv) }} \longrightarrow} \\
& \text { 6, } 62 \%
\end{aligned}
$$

To gain insight into the mechanism, a cross-over experiment was performed (Scheme 3). In the reaction of $1 \mathrm{a}$ and $\mathrm{PhI}(\mathrm{OAc})_{2}$ (1.2 equiv.), butyric anhydride (1.2 equiv.) was added. As a result, in addition to the formation of $\mathbf{2 a}$ in $51 \%$ yield, butyric acid 2-oxo-2-phenyl-ethyl ester (7) was also obtained in $43 \%$ yield. This provides evidence of the liberation of acetic anhydride in the process, which may further participate in the described transformation. In the control experiments, we found that external HOAc (Table 1, Entry 8) had almost no effect on the reaction yield.

Scheme 3 Control experiment.

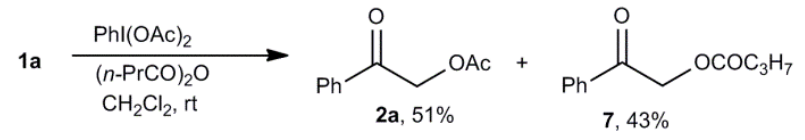

On the basis of the results described above, and along with the previous work by Muñiz, ${ }^{[11,14]}$ Hou, ${ }^{[12]}$ and Ochiai, ${ }^{[20]}$ a plausible mechanism for the conversion of alkynes into $\alpha$-oxygenated ketones is proposed and depicted in Scheme 4. Reaction of the alkyne with $\mathrm{PhI}(\mathrm{OCOR})_{2}$ forms the alkynyliodonium intermediate I. Then, an intermolecular addition of $\mathrm{RCOOH}$ generates vinyliodonium intermediate II. With the liberation of $(\mathrm{RCO})_{2} \mathrm{O}$, the iodonium ylide III is formed, and further electrophilic addition of $(\mathrm{RCO})_{2} \mathrm{O}$ to III gives intermediate IV. ${ }^{[20]} \lambda^{3}$-3-iodane $\mathrm{V}$ is formed via elimination of the acyl cation, followed by protonation. An intramolecular 1,2-acyloxy migration affords the $\alpha$-acyloxy ketone 2 . Hydrolysis of the $\alpha$-trifluoroxy ketone delivers the $\alpha$-hydroxy ketone $\mathbf{3}$ as the final product. The $\mathrm{H}^{+}$source in the IV $\rightarrow \mathrm{V}$ step is supposed to originate from trace amounts of water in the reaction system or directly from the air, considering that the acyl cation liberated is highly moisture-sensitive.

Scheme 4 Proposed mechanism for the formation of $\alpha$-oxygenated ketones $\mathbf{2}$ and $\mathbf{3}$.

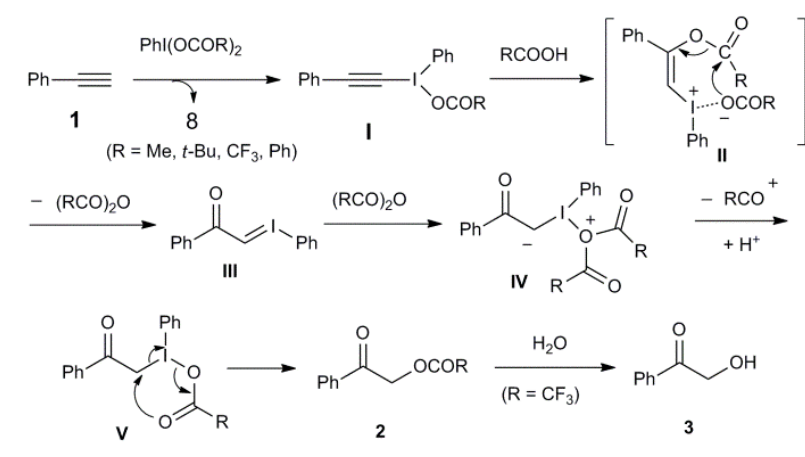

\section{Conclusions}

In summary, we have developed an extremely facile and efficient method for synthesis of $\alpha$-acyloxy ketones and $\alpha$-hydroxy ketones. Terminal alkyne substrates are functionalized with two $\mathrm{C}-\mathrm{O}$ bonds using iodobenzene dicarbonate, avoiding the introduction of a transition-metal catalyst. The reaction is practical, highly atom-efficient and uses mild conditions.

\section{Acknowledgement}

The authors thank the Doctoral Research Foundation of Liaoning Province (No. 20131091), the Postdoctoral Research Foundation of Jilin Province (No. RB201329), and the Collegiate Innovating \& Training Project of the Liaoning Province (2015).

\section{References}

[1] Chen, B.-C.; Zhou, P.; Davies, F.; Ciganek, A. E. Org. React. 2003, 62, 1 .

[2] Adam, W.; Fell, R. T.; Stegmann, V. R.; Saha-Möller, C. R. J. Am. Chem. Soc. 1998, 120, 708.

[3] Morikawa, K.; Park, J.; Andersson, P. G.; Hashiyama, T.; Sharpless, K. B. J. Am. Chem. Soc. 1993, 115, 8463. 
[4] Bøgevig, A.; Sundén, H.; Córdova, A. Angew. Chem., Int. Ed. 2004, 43, 1109.

[5] Nakamura, K.; Kondo, S.-I.; Kawai, Y.; Hida, K.; Kitano, K.; Ohno, A. Tetrahedron: Asymmetry 1996, 7, 409.

[6] Zhdankin, V. V.; Stang, P. J. Chem. Rev. 2008, 108, 5299.

[7] Li, Y.; Song, D.; Dong, V. M. J. Am. Chem. Soc. 2008, 130, 2962.

[8] Kang, Y.-B.; Gade, L. H. J. Org. Chem. 2012, 77, 1610.

[9] Kita, Y.; Yakura, T.; Terashi, H.; Haruta, J.; Tamura, Y. Chem. Pharm. Bull. 1989, 37, 891.

[10] Moriarty, R. M.; Vaid, R. K.; Koser, G. F. Synlett 1990, 7, 365.

[11] Souto, J. A.; Becker, P.; Iglesias, Á.; Muñiz, K. J. Am. Chem. Soc. 2012, 134, 15505.

[12] Mo, D.-L.; Dai, L.-X.; Hou, X.-L. Tetrahedron Lett. 2009 , 50,5578

[13] Ghosh, N.; Nayak, S.; Sahoo, A. K. J. Org. Chem. 2011, 76, 500 .

[14] Souto, J. A.; Martínez, C.; Velilla, I.; Muñiz, K. Angew
Chem., Int. Ed. 2013, 52, 1324.

[15] CCDC 938429 (3e) and 938430 (5) contain the supplementary crystallographic data for this paper. The data can be obtained free of charge from The Cambridge Crystallographic Data Centre via www.ccdc.cam.ac.uk/data_request/ cif.

[16] Fiori, K. W.; Bois, J. D. J. Am. Chem. Soc. 2007, 129, 562.

[17] Kano, T.; Mii, H.; Maruoka, K. J. Am. Chem. Soc. 2009, 131,3450 .

[18] Ueno, M.; Nabana, T.; Togo, H. J. Org. Chem. 2003, 68, 6424.

[19] For the possible mechanism for the formation of $\mathbf{6}$, see the SI.

[20] Ochiai, M.; Kunishima, M.; Fuji, K.; Nagao, Y. J. Org. Chem. 1989, 54, 4038.

[21] Antos, A.; Elemes, Y.; Michaelides, A.; Nyxas, J.; Skoulika, A.; Hadjiarapoglou, S. L. P. J. Org. Chem. 2012, 77, 10949. 